\title{
ENDMEMBER EXTRACTION FOR HYPERSPECTRAL IMAGES USING WATERSHED AND NORMALIZED CUTS
}

\author{
Han $\mathrm{Xu}^{\mathrm{a}}{ }^{*}$, Bangsen Tian ${ }^{\mathrm{b}}$, Fang $\mathrm{Liu}^{\mathrm{c}}$ \\ ${ }^{a}$ College of Resource Environment and Tourism, Capital Normal University, Beijing, China- xuhan2009@gmail.com \\ ${ }^{\mathrm{b}}$ Center for Earth Observation and Digital Earth Chinese Academy of Sciences No.9 Dengzhuang South Road, Haidian \\ District, Beijing 100094, China bstian@ceode.ac.cn \\ ${ }^{\mathrm{c}}$ C\#416 Office, P.O.Box 9718, No. 20 Datun Road, Olympic Science \& Technology Park of CAS, Beijing 100101, China \\ liufang@irsa.ac.cn
}

KEY WORDS: remote sensing images segmentation; watershed transform; normalized cut; endmember extraction; spatial-spectral; hyperspectral data

\begin{abstract}
:
Endmember extraction integrated with spatial information has been concerned on some research recently. In this paper we studies an improved endmember extraction method with spatial preprocessing module which use watershed with normalized cuts to avoid oversegmentation and produce accurate results from spectral mixture analysis. The spatial-spectral endmember extraction method which used the advantages of image segmentation generates the preliminary results and provides important knowledge for future research.
\end{abstract}

* Corresponding author. 


\section{INTRODUCTION}

Endmember extraction is an important task in linear spectral unmixing for mixed-pixel classification and target detection from hyperspectral image data. Many endmember extraction algorithms have been developed over the past decade, such as the pixel purity index (PPI) [1] [2], the N-FINDR algorithm [3], the vertex component algorithm (VCA) [4], the iterative error analysis algorithm (IEA) [5], manual endmember selection tool (MEST) [6], convex cone analysis (CCA) [7], optical real-time adaptive spectral identification system (ORASIS) [8], iterated constrained endmembers (ICE) [9]. These methods were developed for automatic or semi-automatic extraction of spectral endmembers. However, the algorithms are based on applying the spectral information only for searching image endmember. The spectrumbased algorithms can be further improved if spatial information is incorporated into the endmember extraction. These algorithms are significantly different from spectrum-based methods because of the incorporating the spatial information, for instance the endmember can be extracted from a hyperspectral image.

Some advanced techniques for endmember extraction with incorporating information on the spatial adjacent data have been proposed, including the automatic morphological endmember extraction (AMEE) [10], the spatial spectral endmember extraction (SSEE) [11], spatial preprocessing (SPP) approach, and some spatial preprocessing methods for spectral-based endmember extraction. Automatic morphological endmember extraction (AMEE) algorithm is extended the mathematical morphology and calculated the morphological eccentricity index (MEI). AMEE searches each pixel's neighborhood for the most spectrally pure pixels and most mixed pixels. The AMEE is time consuming for it runs on the entire data cube. SSEE is also a spatial-spectral endmember extraction algorithm, applied singular value decomposition to obtain a set of basis vectors that described most of the spectral variability, and spatial averaging was used to find spectrally similar endmember extraction while the vectors were used as skewers to generate a set of candidate endmember. The limitation of this algorithm was that many parameters need to be tuned to identify endmembers. Some spatial preprocessing modules for endmember extraction were developed recently. Those proposed methods can be used to combine with available endmember extraction algorithms. For example, unsupervised clustering method, watershed transformation method and spatial texture method were all attempted to integrate the spatial information to the process of endmember extraction. These preprocessing methods also can not deal endmember extraction with the hyperspectral image segmentation perfectly. Therefore it is necessary to improve the preprocessing module for endmember extraction with spatial information.

In this paper, we present an improved endmember extraction method with spatial preprocessing module which use watershed with normalized cut [12] to avoid over-segmentation and losing the points relevant to the local maxima in each component. The method takes advantages of guiding the endmember seeking process to spectrally purer and spatially homogeneous areas.

\section{Methodology}

\subsection{Dimension reduction}

A dimensionality reduction transformation is applied in the experiment such as principal component analysis (PCA), minimum noise fraction (MNF) [13], singular value decomposition (SVD) or a recently developed Hysime algorithm to reduce the data dimensionality. In this paper, we use MNF transform for feature reduction.

2.2 Image segmentation with Watershed transformation and Normalized cuts

Watershed transformation is a useful image segmentation, the gradient image is considered a topographic surface. It is flooded from regional minima until the entire image has been flooded and a dam is built between lakes. But the over-segmentation is the main problem of the watershed transform. The problem can be improved through the normalized cut algorithm[12]. As the algorithm is a graph partitioning algorithm and the gradient image can be represented by a weighted neighborhood graph, where a node represents a catchment basin of the regions and weighted edges measure the dissimilarity between pairs of regions according to their intensities, spatial locations and original sizes. The spatial process method is used to deal with the hyperspectral data of feature reduction in order to select a set of representative regions in spatialspectral terms. Spectral clustering has emerged as a new and popular clustering method that used eigenvectors of a matrix derive from the data. Spectral graph theory has evolved and encouraged numerous works on digital image processing domain. Normalized cuts algorithm is a kind of feature-space based segmentation technique and popular accepted in recent years, which is based on the graph partitioning and focus on the global criterion. The algorithm is a method for the division of arbitrary undirected graphs based on the weighted edges. The gradient image can be represented by a weighted neighborhood graph, where one node represents one catchment basin of the regions. Therefore those pixels that are spatially purer than those neighbors are remained at the minima of the catchment basins. Watershed transformation and normalized cuts are used to simplify the graph in a lower number of nodes. And the consequent of watershed transformation segmentation is increased significantly.

We assume graph $G=(V, E, W)$, where $V$ are the nodes of the graph and $E$ are the edges among every pair of nodes, the weight on each edge is represented by $w(i, j)$.

$$
\begin{aligned}
& \operatorname{Ncut}(A, B)=\frac{\operatorname{cut}(A, B)}{\operatorname{asso}(A, V)}+\frac{\operatorname{cut}(A, B)}{\operatorname{asso}(B, V)} \\
& \text { Where } \quad \operatorname{cut}(A, B)=\sum_{u \in A, v \in B} w(i, j),
\end{aligned}
$$$$
\operatorname{asso}(A, V)=\sum_{u \in A, k \in V} w(i, k) \text { is the total connection from }
$$

nodes in the set $A$ with all nodes in $V$. The optimal Ncut value can be solved by the generalized eigenvalue system as shown below.

$$
(D-W) y=\lambda D y
$$

Where $D$ is an $N \times N$ diagonal matrix with $d=\sum_{j} w(i, j)$ on its diagonal, $W$ is an $N \times N$ symmetrical matrix with $w(i, j)$. Because of the first Laplacians matrix smallest eigenvalue is 0 , the second smallest eigenvalue is the real 
valued solution to the normalized cut problem.

We need to define the weights on the edges of $G$ based on the similarity and the distance between pixels. The logical translation of the approach taken for segments as follows:

$$
W_{i j}=e\left(\frac{\left\|I_{i}-I_{j}\right\|^{2}}{\partial_{I}}\right) \cdot e\left(\frac{\left\|X_{i}-X_{j}\right\|^{2}}{\partial_{X}}\right)
$$

Where $I_{i}$ and $I_{j}$ are vectors containing the graylevel values of the pixels $i$ and $j ; X_{i}$ and $X_{j}$ are vectors containing the spatial location of these pixels.

\subsection{Endmember extraction with the spatial preprocessing}

The segmentation result produce a set of continuous partitions and the pixels located at the minima of the catchment basins are expected purer than its neighbors. The typical feature endmember can be selected through comparison the endmember candidates and the spectral library. The preprocessing stage is then adopted a spectrum-based endmember extraction algorithm to obtain a set of spatial-spectral representative endmember pixels, and the endmemeber extraction algorithms such as N-FINDR, OSP or VCA can be used in this step.

\section{EXPERIMENTAL RESULT}

The experimental data contains a small segment of a hyperspectral Airborne Visible Infra-Red Imaging Spectrometer (AVIRIS) data set collected in June 1992 over an area in northwestern Indiana ${ }^{1}$ with dimension $145 \times 145$ and the data has 220 bands.

We applied watershed transformation to segment the original image. The number of image segmentation region was 1195 and endmember candidate were showed on figure 1(b). The result presented over-segmentation using watershed transformation. The second watershed transformation was used to decrease the region number and the result was show on figure 1(c). The number of the endmember candidates was significantly reduced to 98 . In the sequence, normalize cut algorithm was integrated into watershed transformation and the number of endmember candidates was reduced to 30 . The result was shown on figure $1(\mathrm{~d})$. The endmember candidates were compared with the spectral library and the typical pure endmembers were selected to unmix the hyperspectral image.

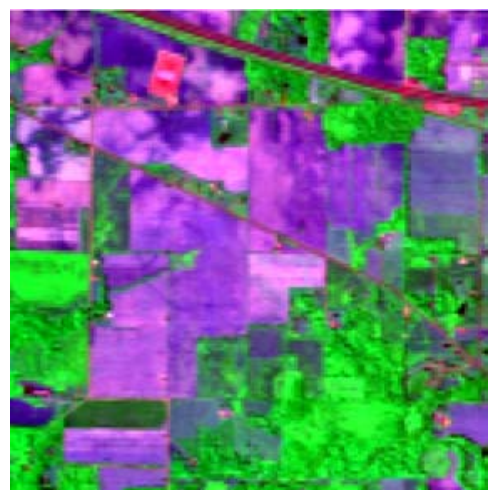

Figure.1. (a) The false color composition of original image

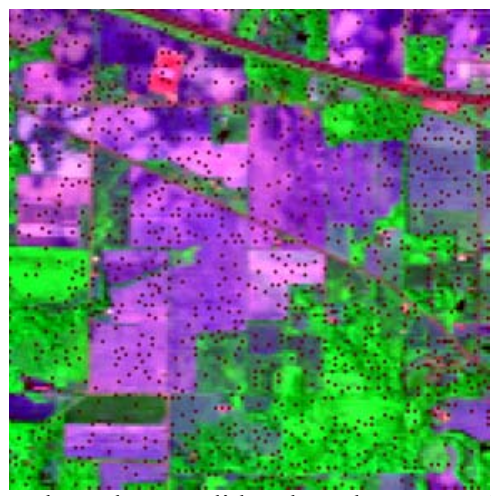

(b) The endmember candidate based on watershed transformation

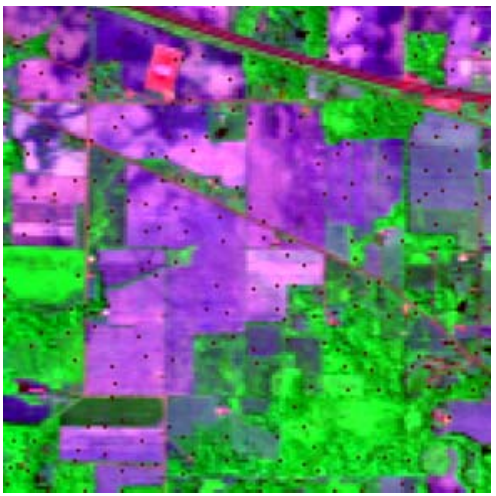

(c) The endmember candidate based on the second watershed transformation

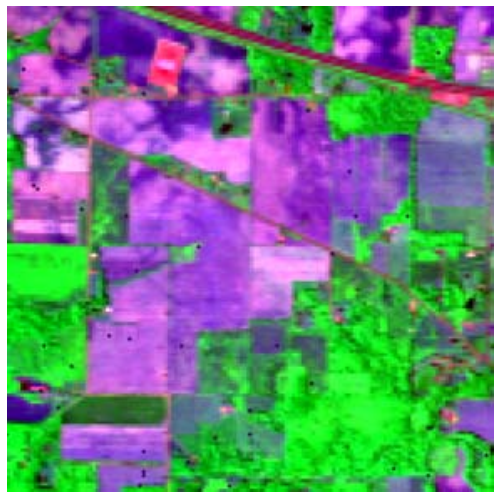

(d) The endmember candidate based on the second watershed 
transformation and Ncut

\section{CONCLUSION}

Based on the analysis aforementioned, an improved method has been developed for endmember extraction in this paper, using watershed transformation with normalized cuts. The proposed algorithm can decrease the over-segmentation of using watershed transformation and emphasize the watershed lines, also prevent losing the points relevant to the local maxima in each component which used watershed transformation with normalized cuts. The experiment results show that the proposed method can improve the endmember extraction which integrated the spatial information. And further experiments on enhance the fractional abundance accuracy should be conducted.

\section{REFERENCES}

[1]Boardman, J. W. (1993). Automating spectral unmixing of AVIRIS data using convex geometry concepts. Summaries of the fourth annual JPL airborne geoscience workshop. JPL Publication 93-26, (pp. 11-14, Vol. 1).

[2]Boardman, J. W., Kruse, F. A., \& Green, R. O. (1995). Mapping target signatures via partial unmixing of AVIRIS data. Summaries, fifth JPL airborne earth science workshop. JPL Publication 95-1, (pp. 23-26, Vol. 1).

[3]Winter, M. E. (1999). Fast autonomous spectral endmember determination in hyperspectral data. Proceedings of the thirteenth international conference on applied geologic remote sensing (pp. 337-344). Vancouver, B.C., Canada, II: ERIM International LTD.

[4]Nascimento, J. M. P., \& Dias, J. M. B. (2005). Vertex component analysis: a fast algorithm to unmix hyperspectral data. IEEE Transactions on Geoscience and Remote Sensing, 43, 898-910.

[5]Neville, R. A., Staenz, K., Szeredi, T., Lefebvre, J., \& Hauff, P.
(1999, 21-24 June). Automatic endmember extraction from hyperspectral data for mineral exploration. Fourth international airborne remote sensing conference and exhibition / 21st Canadian symposium on remote sensing. Ottawa, Ontario,Canada: Natural Resources Canada.

[6]Bateson, A., \& Curtiss, B. (1996). A method for manual endmember selection and spectral unmixing. Remote Sensing of Environment, 55, 229-243.

[7]Ifarraguerri, A., \& Chang, C. -I. (1999). Multispectral and hyperspectral image analysis with convex cones. IEEE Transactions on Geoscience and Remote Sensing, 37, 756-770. [8]Bowles, J., Palmadesso, P. J., Antoniades, J. A., Baumback, M. M., \& Rickard, L. J. (1995). Use of filter vectors in hyperspectral data analysis. Proceedings SPIE infrared spaceborne remote sensing III (pp. 148-157).

[9]Berman, M., Kiiveri, H., Lagerstrom, R., Ernst, A., Dunne, R., \& Huntington, J. F. (2004). ICE: A statistical approach to identifying endmembers in hyperspectral images. IEEE Transactions on Geoscience and Remote Sensing, 42, 2085-2095.

[10]Plaza, A., Martinez, P., Perez, R., \& Plaza, J. (2002). Spatial/spectral endmember extraction by multidimensional morphological operations. IEEE Transactions on Geoscience and Remote Sensing, 40, 2025-2041.

[11]D. M. Rogge, B. Rivard, J. Zhang, A. Sanchez, J. Harris, and J. Feng, "Integration of spatial-spectral information for the improved extraction of endmembers," Remote Sens. Environ., vol. 110, no. 3, pp. 287-303, Oct. 2007.

[12]J. Shi, J. Malik. Normalized cuts and image segmentation. IEEE Transactions on Pattern Analysis and Machine Intelligence PAMI, vol. 22, no. 8, pp. 888-905, 2000.

[13]J. A. Richards, X. Jia, Remote Sensing Digital Image Analysis: An Introduction, Springer, 2006.

${ }^{1}$ Available online: ftp://ftp.ecn.purdue.edu/biehl/PCMultiSpec 